\title{
Predictors of Insufficient Preconception Multivitamin Use: An IMPLICIT Network Study
}

Ramey Roppel, MD | Frank D'Amico, PhD | Ketian Cui, MS | Sukanya Srinivasan, MD, MPH

PRiMER. 2020;4:9.

Published: 5/27/2020 | DOI: 10.22454/PRiMER.2020.832898

\section{Abstract}

Introduction: Adequate maternal nutrition before pregnancy is important to reduce the risk of poor birth outcomes. However, patients report suboptimal intake of multivitamins with folic acid (MVIs).

Methods: We conducted a quality improvement study to identify predictors of insufficient multivitamin use in women of childbearing age at five University of Pittsburgh Medical Center (UPMC) family health centers that implemented the IMPLICIT interconception care (ICC) model of maternal health screenings during well-child visits (WCVs). We derived this analysis from a retrospective chart review of patient-reported demographic information and physician documented maternal behaviors of 758 women who accompanied their children to 2,706 total well-child visits. Insufficient multivitamin use was defined as having one or more visits where the mother reported that she was not taking multivitamins.

Results: Insufficient multivitamin use at these health centers was associated with younger age (OR 0.96, 95\% $\mathrm{Cl} 0.92,0.98)$, less than high school education (OR 3.3, 95\% $\mathrm{Cl} 1.56-6.80)$, public insurance (OR $1.56,95 \% \mathrm{Cl}$ 1.05-2.34), and increased number of well-child visits attended (OR 1.46, 95\% $\mathrm{Cl} 1.31-1.61$ ).

Conclusion: Among women who received screening, younger, low-income, and less educated women are likely to benefit from targeted interventions to improve multivitamin use during the interconception period. Findings also suggest that WCVs are a viable access point to assess and address multivitamin use and other desired maternal health behaviors.

\section{Introduction}

Optimal maternal health and nutrition before pregnancy is important to reduce the risk of poor birth outcomes. ${ }^{1,2}$ However, $45 \%$ of pregnancies in the United States are unintended and therefore women receive no preconception care. ${ }^{3}$ The interval between pregnancies (the interconception period), can serve as a critical time for women to rebuild nutrient resources.

The University of Pittsburgh Medical Center (UPMC) family health centers (FHCs) are members of the IMPLICIT Network, and implemented an interconception care (ICC) model that delivers maternal screenings during well-child visits (WCVs). ${ }^{4}$ Women regularly attend WCVs with their children and are receptive to inquiry from their child's health care provider. ${ }^{5,6}$ Clinicians can use this opportunity to address interconception health risks such as lack of multivitamin with folic acid (MVIs) use. ${ }^{7}$

Regular use of MVIs can potentially reduce the risk of congenital anomalies, increase fetal growth, and benefit infant birth weight. ${ }^{1,-11}$ Despite the possible benefits of MVIs, prevalence of consistent preconception use in US women is 
low and variable. The Pregnancy Risk Assessment Monitoring System estimated national prevalence of preconception multivitamin use was $33.6 \%$ among women aged 18 to 44 years with a recent live birth, and the rate was lowest in younger, Hispanic, non-Hispanic black, and uninsured women. ${ }^{12-13}$ Our study detected predictors of insufficient multivitamin use in women by examining their demographics, characteristics, and behaviors in order to enable providers to identify those at high risk.

\section{Methods}

This study is a retrospective chart review of patient-reported demographic information and clinician-documented maternal behavior, collected as part of IMPLICIT ICC screening from five UPMC FHCs distributed across the metropolitan neighborhoods of Pittsburgh, Pennsylvania. These FHCs are staffed by an interdisciplinary team of physicians and staff who assist in screenings. The UPMC Quality Review Committee (QRC) granted this project exempt status.

Children attend WCVs at developmental milestones, specifically $1,2,4,6,9,12,15,18$, and 24 months, during the first 2 years of life. Standardized maternal demographic surveys were collected one time. At every WCV that mother was present, health care providers attempted to inquire about current multivitamin use, along with other target risk factors, and documented maternal responses until 26 months of age.

Information about related maternal characteristics including parity, methods of contraception chosen, documented maternal intention and initiation of contraceptive methods and comorbid diagnoses (history of neural tube defects, depression, obesity, seizure disorder, diabetes mellitus type I and II, eating disorders, anxiety disorder) was gathered through chart review. The list of comorbidities included diagnoses already associated with decreased multivitamin intake and conditions or medications that may decrease folate levels. ${ }^{14}$

We used only the first WCV for each mother and baby dyad in statistical analysis to ensure the most complete data set. We considered specific baseline characteristics and behavior risks independent variables. Insufficient MVI use, defined as "having one or more WCVs with maternal report of no multivitamin use," was the primary outcome variable. Sufficient MVI use was defined as "having all WCVs with maternal report of current multivitamin use." We used multiple logistic regression methods to examine the relationships between the independent variables and insufficient multivitamin use.

We tabulated frequencies of insufficient and sufficient MVI use for each characteristic or behavior; we used odds ratios and their $95 \%$ confidence intervals to compare risk of insufficient vitamin usage to the independent characteristics. We used likelihood ratio $\chi^{2}$ to obtain the $P$ values with statistical significance accepted at $P<.05$ level, and we adjusted all analyses for the total number of WCV visits.

\section{Results}

Between February 2012 and January 2015, 905 women had a total of 2,706 WCVs at the health centers; 147 women did not report information about multivitamin intake at any WCVs and were excluded from analyses. Of the remaining women, $65 \%$ had insufficient MVI use as defined by the study. We calculated the percentages of women with insufficient/sufficient MVI use for each characteristic or behavior and their risk of insufficient use (Table 1). WCV totals for each woman ranged from one to 11 visits. A large number $(93 \%)$ of these women had five or fewer screenings.

\section{Discussion}

We analyzed data from 758 women followed over 26 months at UPMC FHCs to identify characteristics and behaviors of insufficient MVI use. Women who receive ICC screenings have a slightly higher rate of multivitamin use between pregnancies than the national average, and insufficient use is associated with similar demographics as prior studies. 
The strongest association with insufficient multivitamin use is in patients with less than high school education. When combined with education level, younger age (less than age 25 years) may represent a key target group. These two factors are especially important since younger women (age 18-24 years) have high rates of unintended pregnancy but are least knowledgeable $(6 \%)$ of all age groups about the benefits of folic acid. ${ }^{15}$ Women who qualify for public insurance are also an important target group as a large part of the FHCs population.

Insufficient multivitamin use is associated with more WCVs, due to the strict definition of insufficient use in this study. Women who attended more WCVs may report no MVIs use at least one time, thus become coded as "insufficient," consistent with the clinical observation that patient behaviors change over time. Since $33 \%$ of women reported learning about MVIs from their providers, every opportunity to educate and encourage continued use is critical. $^{15}$

No difference in MVIs use was noted between women with no contraception use versus women with any form of contraception. Since most women stop taking folic acid because they did not intend on becoming pregnant again, lack of MVI use among nonusers of contraception is concerning and deserves attention by clinicians. ${ }^{3,16}$

Limitations of this study include incomplete demographic information, inability to access information from medical records, and inconsistencies in performing and documenting ICC screening results. A limitation of the analysis is the possible changes over time in maternal characteristics that were only assessed at the first WCV. For example, over the same visits that a mother reports not using multivitamins, she may also have changed her choice of contraception.

In conclusion, WCVs are an excellent opportunity to encourage multivitamin use and other healthy behaviors before pregnancy. Younger, less educated and women on public insurance are likely to benefit from targeted interventions to improve multivitamin use during the interconception period.

\section{Tables and Figures}


Table 1: Association of Maternal Characteristics and Behaviors With Insufficient Multivitamin Use

\begin{tabular}{|c|c|c|c|c|c|}
\hline $\begin{array}{l}\text { Maternal Characteristic } \\
\text { or Behavior }\end{array}$ & $\begin{array}{l}\text { Insufficient Use, } n \\
(\%) \text { or Mean (SD) }\end{array}$ & $\begin{array}{l}\text { Sufficient } \\
\text { Use, } \mathbf{n}(\%) \text { or } \\
\text { Mean (SD) }\end{array}$ & $\begin{array}{c}\text { Rate of } \\
\text { Insufficient } \\
\text { Use }\end{array}$ & $\mathrm{OR}^{*}(95 \% \mathrm{Cl})$ & $P$ Value \\
\hline Total visits & 2.6 (SD 1.50) & $3.6(\mathrm{SD} 1.9)$ & -- & $1.46(1.31,1.61)^{1}$ & .001 \\
\hline $\begin{array}{l}\text { Age at incident child's birth ( }<25 \\
\text { years) }\end{array}$ & 26.6 years (SD 5.8 ) & $\begin{array}{l}25.4 \text { years (SD } \\
5.3)\end{array}$ & --- & $0.96(0.92,0.98)^{2}$ & .002 \\
\hline \multicolumn{6}{|l|}{ Race } \\
\hline White & $163(35 \%)$ & $87(36 \%)$ & 0.65 & $0.93(0.53,1.62)$ & .78 \\
\hline Black or African American & $250(54 \%)$ & $131(54 \%)$ & 0.66 & $0.85(0.49,1.45)$ & .54 \\
\hline Other & $53(11 \%)$ & $25(10 \%)$ & 0.68 & Reference & --- \\
\hline \multicolumn{6}{|l|}{ Ethnicity } \\
\hline Non-Hispanic & $429(97 \%)$ & $225(97 \%)$ & 0.65 & Reference & --- \\
\hline Hispanic & $14(3 \%)$ & $7(3 \%)$ & 0.67 & $1.12(0.43,2.94)$ & .81 \\
\hline \multicolumn{6}{|l|}{ Highest Level of Education } \\
\hline Less than high school & $62(20 \%)$ & $11(8 \%)$ & 0.85 & $3.30(1.56,6.80)$ & .001 \\
\hline Attained high school diploma & $111(37 \%)$ & $60(45 \%)$ & 0.65 & $1.02(0.64,1.60)$ & .97 \\
\hline Beyond high school & $130(43 \%)$ & $61(46 \%)$ & 0.68 & Reference & -- \\
\hline \multicolumn{6}{|l|}{ Insurance Type } \\
\hline Public insurance & $363(80 \%)$ & $173(75 \%)$ & 0.68 & $1.56(1.05,2.34)$ & .027 \\
\hline Private or self-pay & $88(20 \%)$ & $58(25 \%)$ & 0.60 & Reference & --- \\
\hline \multicolumn{6}{|l|}{ Smoking History } \\
\hline Never smoked & $192(49 \%)$ & $110(53 \%)$ & 0.64 & Reference & -- \\
\hline Former smoker & $60(15 \%)$ & $34(16 \%)$ & 0.63 & $0.91(0.87,1.93)$ & .68 \\
\hline Currently smoking & $138(35 \%)$ & $64(31 \%)$ & 0.68 & $1.30(0.88,1.92)$ & .19 \\
\hline \multicolumn{6}{|l|}{ Depression Screening } \\
\hline Negative & $343(87 \%)$ & $182(87 \%)$ & 0.65 & $1.12(0.67,1.88)$ & .66 \\
\hline Positive & $50(13 \%)$ & $28(13 \%)$ & 0.64 & Reference & -- \\
\hline \multicolumn{6}{|l|}{ Initial Contraception Method } \\
\hline Not using contraception & $110(28 \%)$ & $60(30 \%)$ & 0.65 & Reference & -- \\
\hline SARC & $222(56 \%)$ & $112(57 \%)$ & 0.67 & $0.99(0.66,1.48)$ & .952 \\
\hline LARC and sterilization & $61(16 \%)$ & $25(13 \%)$ & 0.71 & $1.36(0.76,2.44)$ & .3 \\
\hline Parity including incident child & $2.3(\mathrm{SD} 1.6)$ & $2.1(\mathrm{SD} 1.3)$ & -- & $0.95(0.82,1.06)^{3}$ & .37 \\
\hline Late pregnancy BMI & 32.7 (SD 7.6) & 33.1 (SD 7.7) & -- & $1.02(0.99,1.04)^{4}$ & .251 \\
\hline
\end{tabular}

* All odds ratios are adjusted for number of WCVs.

$1,2,3,4$ The OR is the change in the odds of being insufficient per (1) visit, (2) year, (3) child, (4) BMI point

Abbreviations: SARC, short acting reversible contraception; LARC, long acting reversible contraception; BMI, body mass index; WCV, well-child visit.

\section{Acknowledgments}

This study was presented at the 2017 Annual Family Medicine Education Consortium (FMEC) Meeting, in Cleveland, Ohio.

\section{Corresponding Author}

Sukanya Srinivasan, MD, MPH

McKeesport Family Medicine Residency; 2347 Fifth Avenue, Mckeesport, PA, 15132. 412-673-5504. Fax:

412-673-2150.

srinivasans@upmc.edu 


\section{Author Affiliations}

Ramey Roppel, MD - St. Margaret Family Medicine Residency, University of Pittsburgh Medical Center, Pittsburgh, PA Frank D'Amico, PhD - St. Margaret Family Medicine Residency Program, University of Pittsburgh Medical Center, Pittsburgh, PA

Ketian Cui, MS - Duquesne University, Pittsburgh, PA

Sukanya Srinivasan, MD, MPH - McKeesport Family Medicine Residency | University of Pittsburgh Medical Center, Pittsburgh, PA

\section{References}

1. Dean S V, Lassi ZS, Imam AM, Bhutta ZA. Preconception care: Nutritional risks and interventions. Reprod Health. 2014/11/22. 2014;11:S3.

2. Lu MC, Kotelchuck M, Culhane JF, Hobel CJ, Klerman L V, Thorp Jr. JM. Preconception care between pregnancies: the content of internatal care. Matern Child Heal J. 2006/07/04. 2006;10(5 Suppl):S107-22. https://doi.org/10.1007/s10995-006-0118-7

3. Finer LB, Zolna MR. Declines in unintended pregnancy in the United States, 2008-2011. N Engl J Med. 2016;374(9):843-52.

4. Srinivasan S, Schlar L, Rosener SE, et al. Delivering interconception care during well-child visits: an IMPLICIT Network study. J Am Board Fam Med. 2018;31(2):201-10.

5. Rosener SE, Barr WB, Frayne DJ, Barash JH, Gross ME, Bennett IM. Interconception care for mothers during well-child visits with family physicians: an IMPLICIT Network study. Ann Fam Med. 2016;14(4):350-5. https://doi.org/10.1370/afm.1933

6. Salganicoff A, Ranji U, Wyn R. Women and health care: a national profile - report. San Francisco: The Henry J. Kaiser Family Foundation; 2005. https://kaiserfamilyfoundation.files.wordpress.com/2013/01/women-andhealth-care-a-national-profile-key-findings-from-the-kaiser-women-s-health-survey.pdf. Accessed May 20, 2020.

7. March of Dimes. FMEC IMPLICIT Network. IMPLICIT interconception care toolkit. 2016. https://www.marchofdimes.org/professionals/implicit-interconception-care-toolkit.aspx. Accessed May 2020.

8. Grieger JA, Clifton VL. A review of the impact of dietary intakes in human pregnancy on infant birthweight. Nutrients. 2014;7(1):153-78. https://doi.org/10.3390/nu7010153

9. Ingrid Goh Y, Bollano E, Einarson TR, Koren G. Prenatal multivitamin supplementation and rates of congenital anomalies: a meta-analysis. J Obs Gynaecol Can. 2006;28(8):680-9.

10. Centers for Disease Control and Prevention. Recommendations for the use of folic acid to reduce the number of cases of spina bifida and other neural tube defects. MMWR Recommendations and Reports. Vol. 41. 1992. https://www.cdc.gov/mmwr/preview/mmwrhtml/00019479.htm. Accessed May 20, 2020.

11. Ramakrishnan U, Grant F, Goldenberg T, Zongrone A, Martorell R. Effect of women's nutrition before and during early pregnancy on maternal and infant outcomes: a systematic review. Paediatr Perinat Epidemiol. 2012;26(26)(suppl 1):285-301. https://doi.org/10.1111/j.1365-3016.2012.01281.x

12. Robbins C, Boulet SL, Morgan I, et al. Disparities in preconception health indicators - behavioral risk factor surveillance system, 2013-2015, and pregnancy risk assessment monitoring system, 2013-2014. MMWR Surveillance Summaries. Vol. 67. 2018. https://www.cdc.gov/mmwr/volumes/67/ss/ss6701a1.htm. Accessed May 20, 2020.

13. Srinivasan S, Schlar L, Rosener SE, et al. Delivering interconception care during well-child visits: an IMPLICIT Network study. J Am Board Fam Med. 2018;31(2):201-10.

14. Kennedy $D$, Koren $G$. Identifying women who might benefit from higher doses of folic acid in pregnancy. Can Fam Physician. 2012/04/14. 2012;58(4):394-7.

15. Centers for Disease Control and Prevention. Use of supplements containing folic acid among women of childbearing age--United States, 2007. MMWR. Vol. 57. 2008. https://www.cdc.gov/mmwr/preview /mmwrhtml/mm5701a3.htm. Accessed May 20, 2020.

16. Goldberg BB, Alvarado S, Chavez C. Prevalence of periconceptional folic acid use and perceived barriers to the 
postgestation continuance of supplemental folic acid: survey results from a Teratogen Information Service.

Birth Defects Res A Clin Mol Teratol. 2006;76(3):193-9. https://doi.org/10.1002/bdra.20239

Copyright $@ 2020$ by the Society of Teachers of Family Medicine 